\title{
Impact of videoconferencing applications on mental health
}

\author{
Harkishan Mamtani, ${ }^{1}$ Ruta Karaliuniene, ${ }^{2} \odot$ Renato de Filippis $^{3} \odot$ and \\ Sachin Nagendrappa ${ }^{4}$ ๑
}

${ }^{1} \mathrm{MBBS}$, Junior Resident

Department of Psychiatry, National Institute of Mental Health and Neurosciences,

Bengaluru, India

${ }^{2} \mathrm{MD}$, Clinic for Psychiatry and Psychotherapy, Elblandklinikum Radebeul, Radebeul, Germany

${ }^{3} \mathrm{MD}$, PhD student, Psychiatry Unit, Department Health Sciences, University Magna Graecia of Catanzaro, Italy

${ }^{4} \mathrm{MD}$, Senior Resident, Department of Psychiatry, National Institute of Mental Health and Neurosciences, Bengaluru, India. Email: nsachin40@gmail.com

Keywords. Fatigue; anxiety: videoconferencing applications: gloom; mental health.

First received 20 Feb 2021 Final revision 11 May 2021 Accepted 21 Jun 2021

\section{doi:10.1192/bji.2021.40}

(c) The Author(s), 2021. Published by Cambridge University Press on behalf of the Royal College of Psychiatrists. This is an Open Access article, distributed under Access article, distributed unde the terms of the Creative Commons Attribution licence (http://creativecommons.org/ licenses/by/4.0/), which permits unrestricted re-use, distribution, and reproduction in any medium, provided the original work is properly cited.
Under the circumstances of the current COVID-19 pandemic, videoconferencing applications (apps) have come into the mainstream across the world. Owing to their easy availability and cost-effectiveness, they are used in personal as well as professional lives to communicate. They have been very helpful for students and professionals to ensure that their routine work did not halt when various countries imposed lockdown rules restricting travel, social gatherings and other measures that resulted in reduced inperson meetings. However, they have their own set of disadvantages, aptly called 'Zoom gloom/fatigue', named after a popular videoconferencing platform. Users are also noted to have anxiety while using these apps. Therefore, immediate attention is warranted to ensure cautious usage and to reduce the distress associated with videoconferencing apps while maintaining the obvious advantages that these methods have rapidly spread all over the world.

\section{Benefits of videoconferencing}

Increased usage of digital platforms has become normal, which has also resulted in people's increased digital proficiency. ${ }^{1}$ Videoconferencing applications (apps) are a great example, and one such tool is Zoom. An estimated 10 million people attended Zoom meetings at the end of 2019, and by the end of April 2020 the number rose sharply, to 300 million. ${ }^{2}$ These apps undoubtedly have a multitude of advantages. Indeed, they have ensured that education did not come to a halt. Students around the world continued to learn through informative webinars, and in times when 'physical distancing' was being advocated everywhere, these apps ensured that people socialised with their family and friends using technology, thus preventing the spread of COVID-19.

\section{Adverse effects on mental health}

Despite being useful and having several advantages, numerous concerns have been raised with the increased usage of videoconferencing, more so in the domain of mental health. Many individuals have reported mental tiredness and anxiety resulting from the overuse of these apps, which is being commonly called 'Zoom fatigue' or 'Zoom gloom'.,3 Extra efforts, such as multi-tasking during videoconferencing, can result in mental exhaustion. During in-person conversations, our non-verbal communication is as important as our words. However, in a video call, only a part of our body is visible, and hence it is difficult to catch the non-verbal cues that we would expect during a normal conversation. Looking for nonverbal cues can itself be very tiring for a user. Individuals using videoconferencing apps might feel anxious for several reasons, the major one being a preoccupation with their appearance during calls. And since many apps show the user's picture on the screen, prolonged eye contact and the large face of the speaker can be anxietyprovoking. ${ }^{2,4}$ With the showing of numerous faces of people attending the meeting on the screen, the chances of misinterpretation of expressions make the problem even more challenging. Decoding so many faces makes it difficult for the user to understand what is happening, including what is being said by the speaker. Moreover, various technical and connectivity problems and our helplessness in such situations add to our anxiety. In this regard, a study has shown that response delay due to transmission problems in the telephonic system made users feel that the person at the other end was less attentive and less friendly. ${ }^{4}$

The spheres of our life that before COVID-19 were all quite separated, including work, social life and family, are now happening in the same place, employing technology. Technological support is being used to fulfil multiple roles of a person's life, including professional commitments, social relationships, leisure activities and other goals; the boundaries have been complex and blurred. Hence, in a way, it may make us more susceptible to negative feelings. The impact of the current situation on our feelings and how video calls have made us more vulnerable can be understood from an interesting perspective, i. e. the concept of self-complexity: the less complex our cognitive representation of ourselves is, the more extreme will be our mood changes and selfappraisal. ${ }^{5}$ In this case, since various aspects of our lives are being experienced in the virtual world, there is a possibility that we may become more prone to negative feelings. However, this hypothesis needs validation from studies on the use of videoconferencing apps in particular.

\section{Minimising the adverse effects}

We need to be mindful of these implications and take measures to prevent them. One important 
way to do this is to limit the use of video calls to only when they are really necessary. In conditions where a phone call or an email is enough, video calls should be avoided. Avoiding multi-tasking and scheduling specified time to attend online meetings could significantly add to well-being and resilience. Taking short breaks during long meetings can also help. ${ }^{6}$ Fosslien \& Duffy ${ }^{6}$ suggest looking at the camera, instead of looking directly at the faces onscreen. Looking at the camera will help to reduce anxiety due to the absence of nonverbal cues and can give the feel of a more authoritative presence in the videoconference. They also suggest that preparation beforehand, such as ensuring connectivity, and being mindful during the meeting can also alleviate anxiety. Videoconferencing apps have become a necessity in these challenging times and one cannot ignore their importance. However, it is advisable to follow these simple measures, which can help to reduce the distress caused by them.

A recent review ${ }^{7}$ suggested a few modifications to videoconferencing software to overcome the problem of 'Zoom fatigue'. These include hiding the window that shows one's image by default, or hiding it automatically after a few seconds once the users know that they are framed correctly; setting a maximum limit for the size of the face that is visible on the screen; and audioonly Zoom meetings being made the default setting, instead of the video mode. Implementing these might be a good step from the developers' end to ensure that the apps are more mental health-friendly.

Although the adverse mental health implications of videoconferencing apps are immense, the scientific literature concerning these is very scarce. Robust studies are needed to evaluate these implications and the mechanisms underpinning their causation, along with possible measures to prevent them.

\section{Data availability}

Data availability is not applicable to this article as no new data were created or analysed in this study.

\section{Author contributions}

All authors have contributed equally to this manuscript.

\section{Funding}

This research received no specific grant from any funding agency, commercial or not-for-profit sectors.

\section{Declaration of interest}

None.

\section{References}

1 Richter A. Locked-down digital work. Int J Inf Manage 2020; 55 102157.

2 Wiederhold BK. Connecting through technology during the coronavirus disease 2019 pandemic: avoiding "Zoom Fatigue". Cyberpsychol Behav Soc Networking 2020; 23: 437-8.

3 Sklar J. 'Zoom Fatigue' is taxing the brain. Here's why that happens. National Geographic 2020; 24 Apr (https://www.nationalgeographic. com/science/2020/04/coronavirus-zoom-fatigue-is-taxing-the-brainhere-is-why-that-happens/ [cited 12 Sep 2020]).

4 Schoenenberg K, Raake A, Koeppe J. Why are you so slow? Misattribution of transmission delay to attributes of the conversation partner at the far-end. Int ] Hum Comput Stud 2014; 72: 477-87.

5 Linville PW. Self-complexity and affective extremity: don't put al of your eggs in one cognitive basket. Soc Cogn 1985; 3: 94-120.

6 Fosslien L, Duffy MW. How to combat Zoom fatigue. Harv Bus Rev 2020; 29 Apr (https://hbr.org/2020/04/how-to-combat-zoomfatigue [cited 12 Sep 2020)].

7 Bailenson JN. Nonverbal overload: a theoretical argument for the causes of Zoom fatigue. Technol Mind Behav 2021; 2(1): 1-3. 